\title{
An alternative algorithm for regularization of noisy volatility calibration in Finance
}

\author{
Medarhri Ibtissam ${ }^{1,2}$ - Aboulaich Rajae ${ }^{2}$ - Debit Naima ${ }^{3}$ \\ 1 Rabat Superior National School of Mines, \\ 1000 Agdal Rabat, \\ Morocco \\ 2 LERMA, Mohammadia Engineering School, Mohammed \\ V University, Rabat, Morocco \\ Science and Applied Mathematics \\ 4 Lyon 1 University; Camille Jordan Institut, \\ F-69622 Villeurbanne cedex, France. \\ ibtissamme@gmail.com
}

RÉSUMÉ. Cette contribution dans ce papier est une extension des travaux initiés dans [1], qui présente une stratégie pour l'estimation de la volatilité locale.

En raison du principe de la différence de Morozov [6], le problème de la régularisation de Tikhonov introduite dans [7] est reformulé comme un problème de minimisation de l'inégalité des contraintes. Une procédure Uzawa est proposé de remplacer ce dernier par une séquence de problèmes non contraints traités dans la procédure de régularisation Thikonov modifié dans [1]. Des tests numériques confirment la cohérence de l'approche et l'importante accélérer le processus de détermination de la volatilité locale.

ABSTRACT. This contribution is an extension of the work initiated in [1], presenting a strategy for the calibration of the local volatility.

Due to Morozov's discrepancy principle [6], the Tikhonov regularization problem introduced in [7] is understood as an inequality-constrained minimization problem. An Uzawa procedure is proposed to replace this latter by a sequence of unconstrained problems dealt with in the modified Thikonov regularization procedure in [1]. Numerical tests confirm the consistency of the approach and the significant speed-up of the process of local volatility determination.

MOTS-CLÉS : Calibration du Volatilité locale, problème inverse, régularisation de Tikhonov, principe du Morozov, relaxation de Lagrange, Méthode d'Uzawa

KEYWORDS : Local Volatility calibration, Inverse Problems, Tikhonov regularization, Morozov's principle, Lagrangian Relaxation, Uzawa method. 


\section{Introduction}

The local volatility is an extension of the Black-Scholes constant volatility model [2], it permits to explain the volatility of the shares observed in markets. It assumes the volatility parameter is a deterministic function of both underlying asset price and time. Dupire [3] established that the local volatility function can be uniquely derived from European Option prices given the existence of European options with all strikes and maturities.

In this work we propose to solve calibration problem using the modified Tikhonov regularization proposed in $[1,5]$. the novelty of the present paper stands in the use of the Morozov's Discrepancy Principle to overcome the tuning of the regularization parameter. The problem is stated as an inequality-constrained minimization problem, an uzawa procedure is used to replace it by a sequence of unconstrained problems dealt with in the modified Thikonov regularization procedure in [1].

In section 2 of the abstract, we present the mathematical formulation of the calibration problem. In section 3 , we propose a method to determine the regularized local volatility. Finally, in section 4 implementation issues and numerical results are given.

\section{Description of the calibration problem}

The local volatility model, as defined in Lagnado and Osher [5], assumes that the prices $S$ of an underlying asset follows a general diffusion process :

$$
\frac{d S}{S}=\mu d t+\sigma(S, t) d W_{t}
$$

where $\mu$ is the risk-neutral asset return rate, $W_{t}$ is a standard Brownian motion process, and the local volatility $\sigma$ is a deterministic function that may depend on both the asset price $S$ and the time. Let $V\left(S_{0}, 0, K, T, \sigma(S, t)\right)$ denote the theoretical price of an European option with strike $\mathrm{K}$ and maturity $\mathrm{T}$.

The calibration of the local volatility function to the market is to find a local volatility function $\sigma(S, t)$ such that the theoretical option price computed using this volatility function is between the corresponding bid and ask prices for any option, i.e.,

$$
V_{i j}^{b} \leq V\left(S_{0}, 0, K_{i j}, T_{j}, \sigma(S, T)\right) \leq V_{i j}^{a}
$$

$V_{i j}^{a}$ and $V_{i j}^{b}$ denote the bid and ask prices respectively for an option with strike and maturity.

This problem is usually solved by solving the following optimization problem :

$$
\arg \min _{\sigma}\left\{\left\|V\left(S_{0}, 0, K_{i j}, T_{j}, \sigma(S, T)\right)-\widetilde{V_{i j}}\right\|_{2}^{2}\right\}
$$

where $\widetilde{V_{i j}}$ denote the market price of option. The theoretical option price $V\left(S_{0}, 0, K_{i j}, T_{j}, \sigma(S, T)\right)$ satisfies the following Logarithmic Dupire equation :

$$
\frac{\partial U}{\partial T}(y, T)=-q U(y, T)-\left(r-q+\frac{1}{2} \widehat{\sigma}^{2}(y, T)\right) \frac{\partial U}{\partial y}(y, T)+\frac{1}{2} \sigma^{2}(y, T) \frac{\partial^{2} U}{\partial y^{2}}(y, T)
$$


where $r$ is the risk-free continuously compounded interest rate and $q$ is the continuous dividend yield of the asset.

With the boundary condition follows :

$$
\begin{array}{r}
U\left(y, t_{0}\right)=\max \left(S_{0}-e^{y}, 0\right) \quad \text { for } \quad y_{\min } \leq y \leq y_{\max } \\
\lim _{y \rightarrow-\infty} U(y, T)=S_{0} e^{-q\left(T-t_{0}\right)} \quad \text { for } \quad t_{0} \leq T \leq T_{\max } \\
\lim _{y \rightarrow+\infty} U(y, T)=0 \quad \text { for } t_{0} \leq T .
\end{array}
$$

As for most inverse problems, it is ill-posed in the sense that small changes in the option prices may lead to big changes in the volatility function. When noises are included in option prices, which is usually the case in reality, the reconstructed volatility function will be unstable and blow up. In order to overcome the ill-posedness nature of the calibration problem, we propose a Tikhonov regularization in [1]. We seek for $\sigma^{\star}$ solution of minimization problem

$$
\arg \min _{\sigma}\left\{\lambda\left\|V\left(S_{0}, 0, K_{i j}, T_{j}, \sigma(S, T)\right)-\widetilde{V_{i j}}\right\|_{2}^{2}+\|\nabla \sigma\|_{2}^{2}\right\}
$$

\section{Alternative strategy for selection Tikhonov regularization parameter}

In order to avoid an expensive calculation of the regularization parameter in problem 7 as considered in [1], we use a relaxed version of Morozov's discrepancy principle. Problem 7 may be understood as a constrained minimization problem, say

$$
\arg \min _{\sigma}\|\nabla \sigma\|_{2}^{2}
$$

subject to the relaxed constraint (see [4]),

$$
\left\|V\left(S_{0}, 0, K_{i j}, T_{j}, \sigma(S, T)\right)-\widetilde{V_{i j}}\right\|_{2}=\tau \delta
$$

where the parameter $0<\tau<1$ weighs the available information on the noise $\delta$.

The constraint 9 states that the regularized data satisfies

$$
\left\|V\left(S_{0}, 0, K_{i j}, T_{j}, \sigma(S, T)\right)-\widetilde{V_{i j}}\right\|_{2}<\delta
$$

which leads to an inequality-constrained minimization problem.

The uzawa method is a way to replace a constrained minimization problem by a sequence of unconstrained ones. Since these unconstrained problems have been addressed in [1], the method seems to be a natural choice to introduce a significant seep-up in the determination of the regularized volatility.

\section{Algorithm and Numerical Result}

We give here the algorithm to reconstruct the local volatility function. 


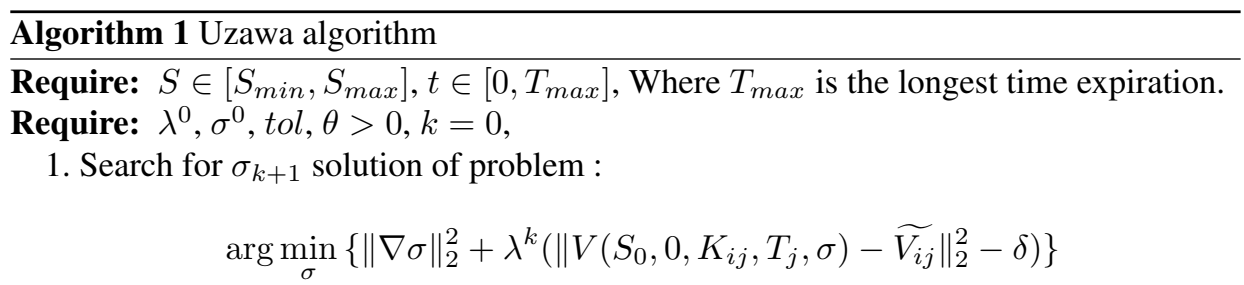

2. Compute the updated value of constraint : $g^{k}:=\left(\| V\left(S_{0}, 0, K_{i j}, T_{j}, \sigma^{k+1}\right)-\right.$ $\left.\widetilde{V_{i j}} \|_{2}^{2}-\delta\right)$

3. $\lambda^{k+1}=\lambda^{k}-\theta g^{k}$

4.

while $g^{k} \geq$ tol do

5. $\mathrm{k}=\mathrm{k}+1$, return to step 1

end while

return $\sigma^{k}$

In step 2 the unconstrained minimization problem is the one addressed in [1]. We recall briefly the procedure adopted there, with the lagrangian

$$
\mathcal{L}(\sigma, \lambda)=\|\nabla \sigma\|_{2}^{2}+\lambda\left(\left\|V\left(S_{0}, 0, K_{i j}, T_{j}, \sigma\right)-\widetilde{V_{i j}}\right\|_{2}^{2}-\delta\right)
$$

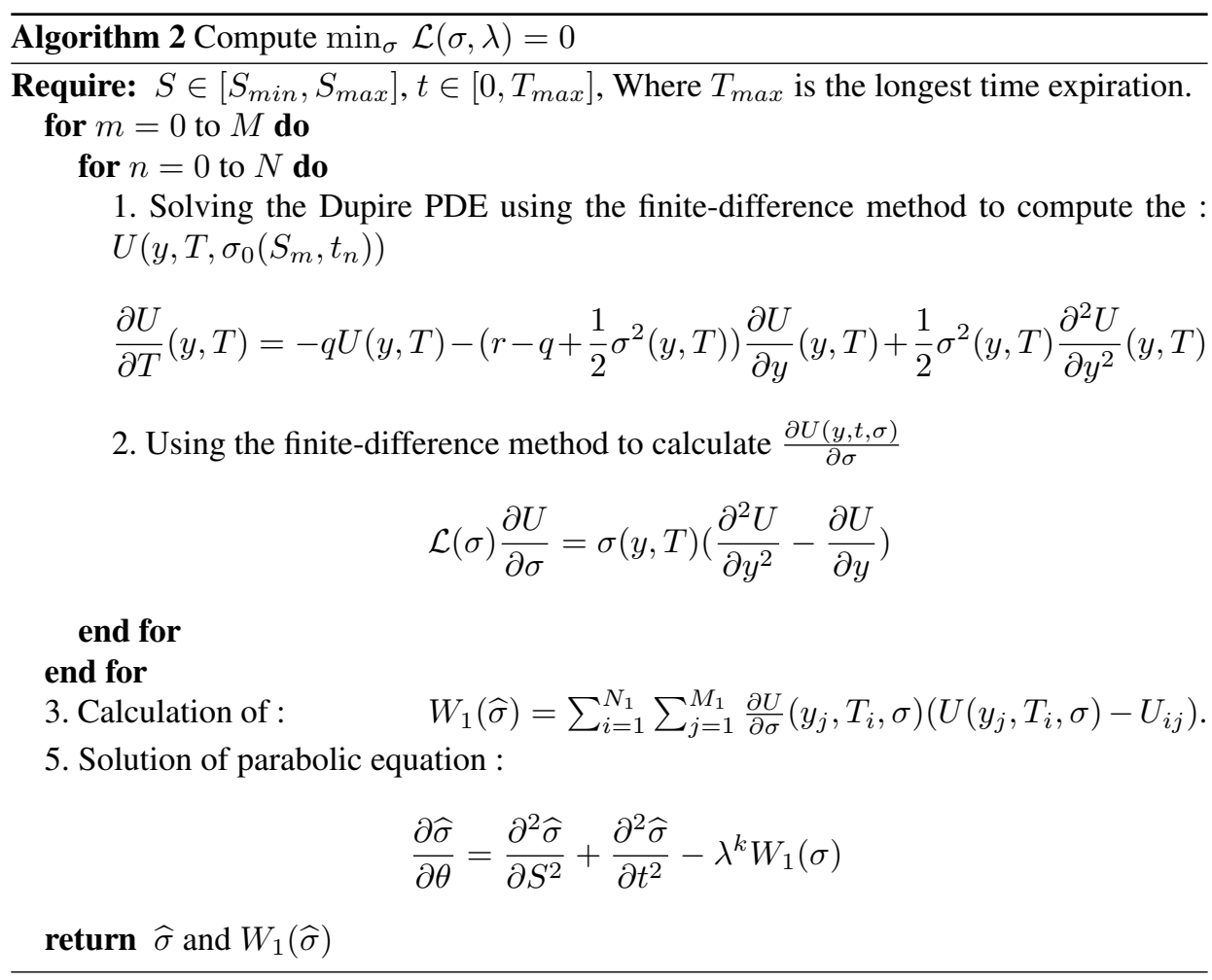

Numerical test : To test this Algorithm we choose the example used in [1]. We consider the true volatility function $\sigma_{\text {true }}(S)=0.2+0.1 e^{-0.2 S}$, we use this function for generate a set of option prices $U^{i j}$ by solving Dupire equation3, the interest rate $r=0.05$ 
we consider $T_{\max }=10$, and the strike $K \in[50,160]$.

For $\lambda^{0}=30$ and $\theta=1 / 2$, we illustrate the different results of the proposed Algorithm :

\begin{tabular}{|c|c|c|c|c|c|c|c|c|}
\hline $\mathrm{i}$ & 1 & 2 & 3 & 4 & 5 & 6 & 7 & 8 \\
\hline Constraint value $g^{i}$ & 0.6882 & 0.2094 & 0.0745 & 0.0193 & 0.0054 & 0.0016 & $5.0 \mathrm{e}-004$ & $8.00 \mathrm{e}-005$ \\
\hline
\end{tabular}

Tableau 1. The results of local volatility with $\lambda^{0}=30$

At iteration 8, the error $\left\|\sigma^{8}-\sigma_{\text {true }}\right\|=1.0430 e-005$, the $\lambda^{*}=35$ and in [1] we obtain after 12 iteration with $\lambda$ fixed at 30 an error $\left\|\sigma_{k}-\sigma_{\text {true }}\right\|=0.21510^{-4}$.

Conclusion : The numerical experiment prove the acceleration convergence. Further convergence history of the algorithm will be discussed relatively to the expected behavior.

\section{Acknowledgement}

Authors thank the Volubilis Hubert Curien Program (MA/13/286) for financial support.

\section{Bibliographie}

[1] Aboulaich R ., Medarhri I., « Calibration of Volatility : Coupling between alternative regularization strategy and Dupire's Equation », Adv. International Journal of Applied Mathematics and Staistics, vol. 42, $\mathrm{n}^{\circ} 12,2013$.

[2] Black F., Scholes M., « The pricing of options and corporate liabilities », Journal of Political Economy, vol. 81, 1973.

[3] DuPIRE B., «Pricing with smile », Risk,vol, vol. 7,n ${ }^{\circ}$ 1, 1994.

[4] Kirsh A., «An Introduction to the Mathematical Theory of Inverse Problems », Applied Mathematical Sciences, vol. 120, ${ }^{\circ}$ 2, 2011.

[5] Lagnado R., Osher S., « Technique for calibrating Derivative Security pricing models :Numerical solution of an inverse problem », The journal of computational Finance, vol. 1, 1997.

[6] Morozov V. A., « Methods for solving incorrectly posed problems (translation ed. : Nashed M. Z.) », Wien, New York : Springer, 1984.

[7] Tikhonov A. N., ARSEnIN V. YA., «Solutions of ill-posed problems », New York, WinstonWiley, 1977. 